\title{
Glutamate supplementation is associated with improved glucose metabolism following carbohydrate ingestion in healthy males
}

\author{
Katie M. Di Sebastiano, Kirsten E. Bell, Tyler Barnes, Anushka Weeraratne, \\ Tahira Premji and Marina Mourtzakis* \\ Department of Kinesiology, University of Waterloo, \\ 200 University Avenue West, Waterloo, ON, Canada N2L $3 G 1$
}

(Submitted 28 September 2012 - Final revision received 5 March 2013 - Accepted 22 April 2013 - First published online 11 June 2013 )

\section{Abstract}

Glutamate is linked to the glycolytic process, particularly when co-ingested with carbohydrate, but its effects on glucose metabolism are poorly characterised. The present study aimed to (1) specifically examine the effects of carbohydrate administration on circulating glutamate concentrations and (2) investigate the effect of increased glutamate availability, independent of carbohydrate ingestion, on glucose metabolism. A total of nine participants underwent four trials: (1) glutamate supplement + carbohydrate drink (GLU + CHO); (2) glutamate supplement + placebo drink (GLU); (3) placebo supplement + carbohydrate drink (CHO); (4) placebo supplement + placebo drink (CON). Following a fasting blood sample, participants ingested monosodium L-glutamate (MSG; $150 \mathrm{mg} / \mathrm{kg}$ body weight) or placebo capsules at each trial followed by a $75 \mathrm{~g}$ carbohydrate or a non-energy placebo drink 30 min later. Blood samples were taken at $0,10,20,30,40,50,60,75,90,105$ and $120 \mathrm{~min}$. Plasma glutamate concentrations were significantly elevated relative to baseline during the GLU (approximately 10-fold) and GLU + CHO trials (approximately 6-fold). The glucose response to a carbohydrate load was blunted when glutamate was increased in the circulation (peak serum glucose: 5.50 (sE 0.54) mmol/l during the GLU + CHO trial $v$. 7.69 ( $\mathrm{se} 0.53) \mathrm{mmol} / \mathrm{l}$ during the $\mathrm{CHO}$ trial, $P<0.05$ ). On average, c-peptide results revealed that insulin secretion did not differ between the $\mathrm{GLU}+\mathrm{CHO}$ and $\mathrm{CHO}$ trials; however, four participants demonstrated increased insulin secretion during the GLU + CHO trial and five participants demonstrated decreased insulin secretion under the same conditions. In conclusion, when administration is staggered, MSG and carbohydrate supplementation can be used to manipulate plasma glutamate; however, future studies should control for this dichotomous insulin response.

\section{Key words: Amino acids: HPLC: Insulin}

Glutamate is the primary amino acid taken up by resting and exercising skeletal muscle ${ }^{(1-3)}$, and it has a role in a variety of metabolic processes. There have been several studies that have examined the role of glutamate in energy metabolism through its interactions with pyruvate, alanine and glutamine ${ }^{(4-7)}$. Despite these links, which suggest a role for glutamate in gluconeogenic processes in visceral tissues and glycolytic processes in both muscle and visceral tissues, the functional significance of glutamate specifically related to glucose metabolism is poorly characterised.

Glutamate supplementation in the form of monosodium L-glutamate (MSG) has been used as a tool to explore the role of glutamate in energy metabolism by increasing available plasma glutamate by $7-18$-fold ${ }^{(4,7,8)}$. When plasma glutamate is augmented to these magnitudes, various components of glucose metabolism are affected $^{(9-11)}$. When MSG was administered to healthy ${ }^{(9)}$ and type 2 diabetic $^{(10)}$ rats during an oral glucose tolerance test, an increase in insulin secretion and improved glucose tolerance were observed compared with no MSG administration. However, in human subjects, simultaneous glutamate and carbohydrate administration has not resulted in improved glucose tolerance ${ }^{(11)}$. It is likely that the protocol used may have confounded these results; circulating glutamate concentrations are attenuated when carbohydrate is co-administered with $\mathrm{MSG}^{(12,13)}$.

The simultaneous co-administration of carbohydrate and MSG delays the time that plasma glutamate concentration peaks as well as the magnitude of this peak ${ }^{(4,11,12)}$. It is possible that, when given simultaneously, carbohydrate enhances the retention of glutamate at the gut via the alanine aminotransferase reaction, which requires pyruvate (from carbohydrate) and glutamate to produce alanine and the

Abbreviations: $\mathrm{CHO}$, placebo supplement + carbohydrate drink; CON, placebo supplement + placebo drink; GLU, glutamate supplement + placebo drink; GLU + CHO, glutamate supplement + carbohydrate drink; MSG, monosodium L-glutamate. 
Krebs cycle intermediate 2-oxoglutarate. However, it is possible that delaying the ingestion of carbohydrate, which requires approximately $10 \mathrm{~min}$ to reach peak plasma concentrations following ingestion ${ }^{(14)}$, relative to the administration of glutamate, which requires approximately $40-60 \mathrm{~min}$ to peak $^{(4)}$, may result in simultaneous peak glutamate and glucose concentrations in the circulation. Presumably, if carbohydrate administration is delayed relative to MSG ingestion, glutamate will not be detained to the same extent in the gut and glutamate will reach higher circulating concentrations. To examine the interactions of glutamate on peripheral glucose metabolism in future studies, it is important to identify a methodological approach that gives rise to simultaneous peak plasma glutamate and glucose concentrations.

The objective of the present study was to examine the potential effects of increased glutamate availability on glucose and insulin metabolism following carbohydrate ingestion. We hypothesised that ingesting carbohydrate 30 min following glutamate administration would result in simultaneous peak plasma glutamate and glucose concentrations. Further, we hypothesised that using this staggered protocol of combining glutamate and carbohydrate supplementation would result in lower peak glucose concentrations and that insulin secretion would be greater compared with carbohydrate alone, implying improved glucose uptake.

\section{Methods}

\section{Participants}

A total of nine healthy, recreationally active males were recruited to participate in the present study. The mean age, body mass and BMI of the participants were 23.9 (SD 1.9) years, $79 \cdot 4(\mathrm{SD} 9 \cdot 0) \mathrm{kg}$ and $25.0(\mathrm{SD} 2 \cdot 4) \mathrm{kg} / \mathrm{m}^{2}$, respectively. Individuals with hypertension, diabetes, cardiac arrhythmias or intolerance to MSG were excluded from the study. The present study was conducted according to the guidelines laid down in the Declaration of Helsinki, and all procedures involving human subjects were approved by the University of Waterloo Research Ethics Board. Written informed consent was obtained from all subjects.

\section{Experimental protocol}

Participants underwent four different trials: (1) glutamate supplement + carbohydrate drink (GLU + CHO); (2) glutamate supplement + placebo drink (GLU); (3) placebo supplement + carbohydrate drink (CHO); (4) placebo supplement + placebo drink (CON). Glutamate supplement was given as MSG in gelatin capsules at a dose of $150 \mathrm{mg} / \mathrm{kg}$ body weight, which has been used in previous studies ${ }^{(4,7,8,12)}$. Placebo supplement was also given in gelatin capsules that contained $\mathrm{NaCl}$ in the same proportions as in the MSG capsules, while dextrose and sucralose were used to match the remaining weight of the capsules. The total amount of dextrose used in placebo capsules was not greater than $3 \mathrm{~g}$ in total. Carbohydrate drinks (Trutol ${ }^{\circledR}$ ) contained $75 \mathrm{~g}$ dextrose and placebo drinks consisted of a similar-tasting, orange-flavoured, non-energy drink.
The trials were conducted in a double-blind, cross-over manner and were separated by 1 week. Trial order was randomly assigned. Participants arrived for each trial after an overnight fast $(8-12 \mathrm{~h})$. For $2 \mathrm{~d}$ before each trial, participants were instructed to (1) refrain from alcohol and exercise, (2) complete a Baecke questionnaire and physical activity diary before the first trial and follow the same activities listed in the diary for the $2 \mathrm{~d}$ before each subsequent trial and (3) record their food intake for $2 \mathrm{~d}$ before the first trial and maintain the same diet $2 \mathrm{~d}$ before each subsequent trial.

Upon arrival for each trial, weight was recorded and a catheter inserted into an antecubital vein of the participant's arm. After drawing a fasting blood sample, the participants ingested MSG or placebo capsules. Plasma glutamate concentration has been shown to peak approximately $50 \mathrm{~min}$ post-MSG ingestion. At $30 \mathrm{~min}$ following ingestion of the capsules, participants ingested a carbohydrate or placebo drink. Petrie et $a l .{ }^{(14)}$ have shown that serum glucose concentration rises within $15 \mathrm{~min}$ and peaks at 20-30 min following carbohydrate ingestion. Therefore, delaying carbohydrate ingestion $30 \mathrm{~min}$ should allow serum glucose concentration to peak approximately $50-60 \mathrm{~min}$ following the ingestion of the capsules. Thus, by staggering the administration of both supplements as we have described, we anticipated that a simultaneous peak in both glucose and glutamate concentrations would be achieved. Blood samples were taken at $0,10,20,30,40,50,60,75,90105$ and $120 \mathrm{~min}$.

\section{Biochemical analyses}

Approximately $8 \mathrm{ml}$ of whole blood were collected at each time point. For analysis of amino acids, blood was treated with heparin, centrifuged and plasma was stored at $-80^{\circ} \mathrm{C}$. Amino acids were analysed using HPLC (Waters 2695 Separations Model) and the picotag method, as described previously $^{(15)}$. The remaining blood for each time point was allowed to clot, centrifuged and serum was stored at $-80^{\circ} \mathrm{C}$ for the analysis of glucose, insulin (Coat-A-Count Insulin RIA Kit; Siemans Healthcare Diagnostics), c-peptide (C-Peptide Double Antibody RIA Kit; Siemans Healthcare Diagnostics), lactate, NEFA (Wako Diagnostics) and glycerol. Glucose, lactate and glycerol were analysed using fluorometric methods, as described previously ${ }^{(16)}$. NEFA were analysed using spectrophotometric methods ${ }^{(17)}$. For glucagon analysis (Glucagon Double Antibody RIA Kit; Siemans Healthcare Diagnostics), blood was treated with EDTA/aprotinin, centrifuged and plasma was stored at $-80^{\circ} \mathrm{C}$.

\section{Calculations}

The AUC for glucose, insulin and c-peptide were calculated using the incremental area method ${ }^{(18)}$. We assessed insulin sensitivity during the $\mathrm{GLU}+\mathrm{CHO}$ and $\mathrm{CHO}$ trials using the index developed by Matsuda \& Defronzo ${ }^{(19)}$. This index provides an estimate of whole-body insulin sensitivity using glucose and insulin values over the course of a $75 \mathrm{~g}$ oral glucose tolerance test and has been validated against the euglycaemic insulin clamp. The sum of all amino acids 
was calculated and referred to as total amino acids. The sum of the essential amino acids threonine, valine, methionine, isoleucine, leucine, phenylalanine, tryptophan and lysine was referred to as total essential amino acids, and the sum of valine, leucine and isoleucine was referred to as total branched-chain amino acids.

\section{Statistics}

All data were analysed using two-way repeated-measures ANOVA (trial $\times$ time point) with the exception of the AUC calculations for c-peptide, glucose and insulin, which were analysed using one-way repeated-measures ANOVA (trial). The Matsuda insulin sensitivity indices for the GLU + CHO and $\mathrm{CHO}$ trials were compared using a paired $t$ test. In a sub-analysis between two groups of participants who demonstrated different insulin responses to the GLU + CHO trial, we compared groups using a Student's $t$ test. Statistical significance was accepted at $P \leq 0.05$ and significant differences revealed using ANOVA were analysed using Tukey's post hoc test. Statistical analysis was performed using SigmaPlot ${ }^{\circledR}$, version 11.2 (Systat Software, Inc.). All data are presented as means with their standard errors.

\section{Results}

\section{Plasma amino acids}

During the GLU trial, plasma glutamate was significantly elevated from baseline at $40 \mathrm{~min}$ and, on average, reached peak concentrations (approximately 10-fold from baseline) by $50 \mathrm{~min}$ (individual ranges for peak concentrations: 40-75 min; Fig. 1). In the GLU + CHO trial, plasma glutamate concentrations were significantly elevated at $30 \mathrm{~min}$ and reached peak concentrations (approximately 6-fold from baseline) earlier than the GLU trial at $40 \mathrm{~min}$ (individual ranges for peak concentrations: $40-50 \mathrm{~min}$; Fig. 1). No changes in plasma glutamate concentrations compared with baseline were observed in the $\mathrm{CHO}$ or $\mathrm{CON}$ trial.

Plasma aspartate concentrations, which closely relates to glutamate metabolism, exhibited an approximately 3-fold increase from baseline during the GLU trial (33.8 (SE 7.8) $v$. 9.9 (SE 2.1) $\mu \mathrm{mol} / 1, \quad P<0.05$ ). Similar to plasma glutamate, aspartate concentrations achieved peak concentrations between 40 and $90 \mathrm{~min}$. During the GLU + CHO trial, an approximately 2-fold increase in plasma aspartate concentrations was observed compared with baseline (26.4 (SE 4.8) v. 11.5 (se 1.4$) \mu \mathrm{mol} / 1, P<0.05$ ). Again, similar to the patterns demonstrated with glutamate concentrations, plasma aspartate concentrations in this trial peaked between 30 and $50 \mathrm{~min}$. No significant changes were observed in plasma alanine, glutamine, branched-chain amino acid, essential amino acid or total amino acid concentrations compared with baseline in any of the experimental conditions. No changes were observed in any of the other individual plasma amino acids (data not presented).

\section{Glucose and insulin metabolism}

The glucose response to a carbohydrate load was blunted when glutamate was increased in the circulation (peak serum glucose: 5.50 (SE 0.54$) \mathrm{mmol} / \mathrm{l}$ during the GLU + CHO trial v. 7.69 (SE 0.53$) \mathrm{mmol} / 1$ during the $\mathrm{CHO}$ trial, $P<0.05)$. During the $\mathrm{CHO}$ trial, serum glucose concentrations peaked between 50 and $105 \mathrm{~min}$ with two-thirds of the participants peaking at $60 \mathrm{~min}$ (i.e. two-thirds of the participants peaked

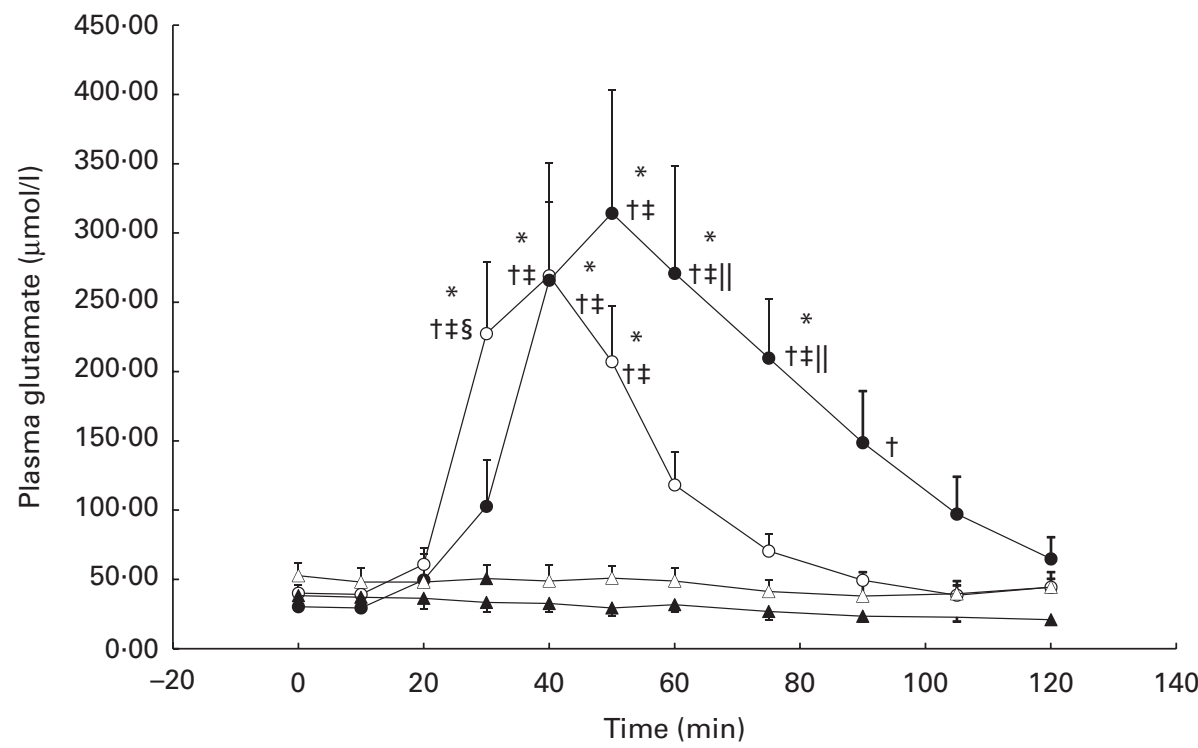

Fig. 1. Plasma glutamate concentrations during the glutamate + carbohydrate (GLU $+\mathrm{CHO} ; \mathrm{O})$, glutamate only (GLU; $\bullet$ ), carbohydrate only ( $\mathrm{CHO} ; \Delta)$ and control (CON; $\Lambda)$ trials. Values are means, with their standard errors represented by vertical bars. ${ }^{\star}$ Mean value was significantly different from fasting values within each trial $(P<0.05)$. † Mean value was significantly different from that of the CON trial $(P<0.05)$. $\ddagger$ Mean value was significantly different from that of the $\mathrm{CHO}$ trial $(P<0.05)$. $§$ Mean value was significantly different from that of the GLU trial $(P<0.05)$. \|Mean value was significantly different from that of the $\mathrm{GLU}+\mathrm{CHO}$ trial $(P<0.05)$. Due to technical problems in blood draws, $n 8$ for time points $75-120$ min in the CON trial. Due to analytical problems, $n 8$ for time point $10 \mathrm{~min}$ in the $\mathrm{GLU}+\mathrm{CHO}$ trial, for time points 105 and $120 \mathrm{~min}$ in the GLU trial and for time point $120 \mathrm{~min}$ in the $\mathrm{CHO}$ trial. 


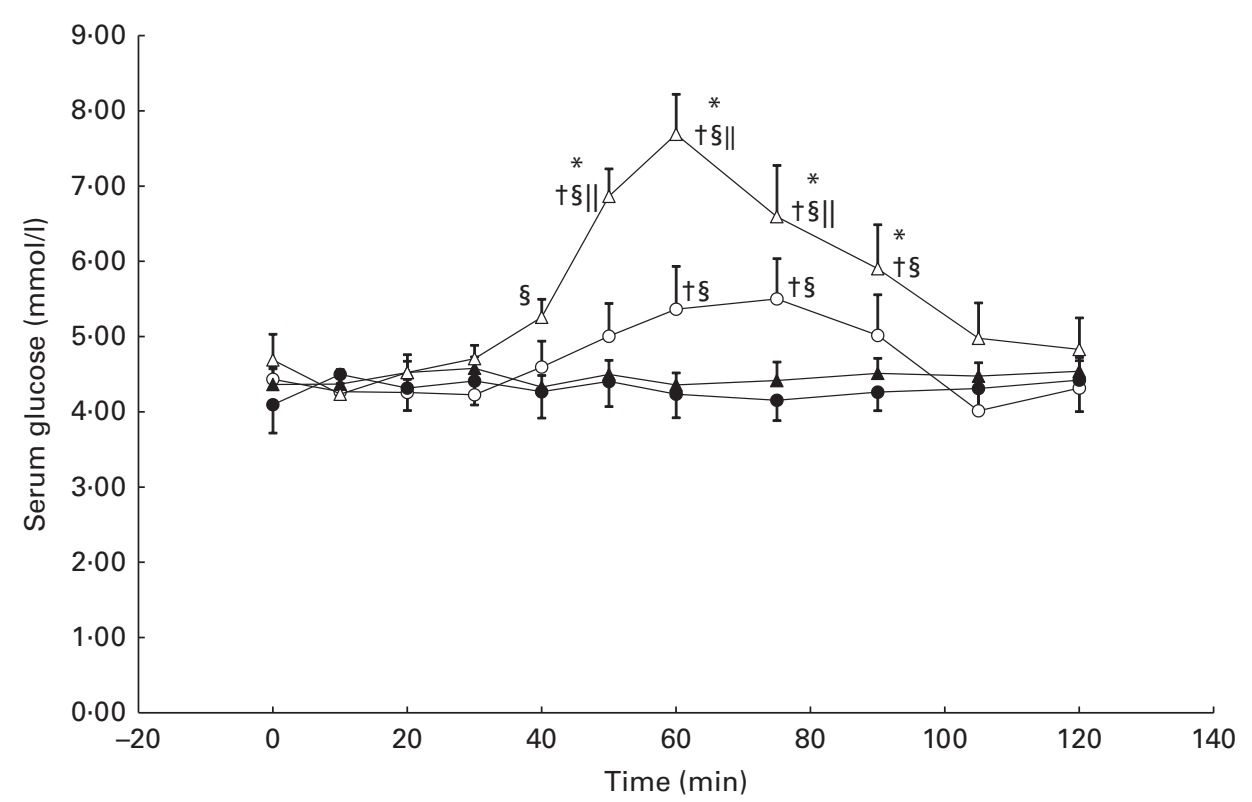

Fig. 2. Serum glucose concentrations during the glutamate + carbohydrate (GLU $+\mathrm{CHO}$; $\mathrm{O})$, glutamate only $(\mathrm{GLU}$; $\bullet$ ), carbohydrate only $(\mathrm{CHO} ; \Delta)$ and control $(\mathrm{CON} ; \mathbf{\Delta})$ trials. Values are means, with their standard errors represented by vertical bars. * Mean value was significantly different from fasting values within each trial $(P<0.05)$. † Mean value was significantly different from that of the CON trial $(P<0.05)$. $\S$ Mean value was significantly different from that of the GLU trial $(P<0.05)$. \|Mean value was significantly different from that of the GLU $+\mathrm{CHO}$ trial $(P<0.05)$. Due to technical problems in blood draws, $n 8$ for time points $75-120 \mathrm{~min}$ in the CON trial.

30 min post-carbohydrate ingestion). Compared with the $\mathrm{CHO}$ trial, the rise in serum glucose concentrations during the GLU + CHO trial was delayed and was not significantly different from baseline despite being statistically different from the CON and GLU trials (Fig. 2). No changes in serum glucose concentrations were observed during the GLU or CON trial. Glucose AUC data are presented in Table 1.

As anticipated, serum insulin concentrations were elevated compared with baseline in response to carbohydrate ingestion during the $\mathrm{CHO}$ and GLU + CHO trials; however, there were no differences in insulin concentrations between these two trials (Fig. 3). C-peptide concentrations followed a similar pattern to insulin concentrations (Fig. 4), where c-peptide concentration was elevated during the $\mathrm{CHO}$ and $\mathrm{GLU}+\mathrm{CHO}$ trials relative to baseline and relative to the GLU and CON trials. Insulin sensitivity was not different between the $\mathrm{CHO}$ and GLU + CHO trials (Matsuda index: 15.45 (SE 1.58) $v$. 15.39 (sE 2.98), $P=0.426$ ). Insulin AUC data are presented in Table 1. Glucagon concentrations were variable across the participants but consistent between the trials for each participant. There were no statistical differences across any time points or between the trials for the glucagon data $(P=0 \cdot 130)$.

The large variability for serum insulin in the GLU + CHO trial when compared with the GLU and CON trials (Fig. 3) prompted us to conduct further analysis. Our sub-analysis revealed that four participants (increased insulin group) demonstrated the hypothesised increase in insulin secretion during the GLU + CHO trial compared with the $\mathrm{CHO}$ trial. On average, these participants experienced a 31 (SE 9) \% increase in insulin AUC during the GLU + CHO trial relative to the $\mathrm{CHO}$ trial (range 14-52\%). However, the remaining five participants (decreased insulin group) demonstrated the opposite effect: insulin secretion was decreased in the $\mathrm{GLU}+\mathrm{CHO}$ trial relative to the $\mathrm{CHO}$ trial. The average decrease in insulin AUC was 33 (SE 13) \% (range 10-85\%) during the GLU + CHO trial compared with the $\mathrm{CHO}$ trial. There was no significant difference between the two groups of participants in physical activity level based on the Baecke sport index (increased insulin group: 2.81 (SE 0.68) $v$. decreased insulin group: 3.56 ( $\operatorname{se} 0 \cdot 19), P=0.278$ ), fasting glucose (increased insulin group: $4.42(\mathrm{SE} 0 \cdot 13) \mathrm{mmol} / \mathrm{l} v$. decreased insulin group: 4.45 (SE 0.24$) \mathrm{mmol} / \mathrm{l}, P=0.915$ ), Matsuda insulin sensitivity index (increased insulin group: 15.91 ( $\operatorname{se} 2.88$ ) $v$. decreased insulin group: 15.09 (SE 1.98), $P=0.816$ ) or plasma glutamate (increased insulin group: 36892 (SE 18883) pmol/l per min $v$. decreased insulin group: 65819 (sE 13202) pmol/1 per min, $P=0 \cdot 236$ ) during the GLU + $\mathrm{CHO}$ trial. In light of this dichotomous response of insulin to glutamate ingestion, caution should be used when interpreting the results of the present study.

Table 1. AUC for glucose and insulin across the trials (Mean values with their standard errors)

\begin{tabular}{lccccc}
\hline & \multicolumn{2}{c}{$\begin{array}{c}\text { Glucose AUC } \\
(\mathrm{mmol} / \mathrm{l} \times \mathrm{min})\end{array}$} & & \multicolumn{2}{c}{$\begin{array}{c}\text { Insulin AUC (pmol/l } \times \\
\text { min })\end{array}$} \\
\cline { 2 - 3 } \cline { 6 - 7 } Trial & Mean & SE & & Mean & SE \\
\hline GLU + CHO & 31 & 35 & & $21619^{*} \dagger$ & 2016 \\
GLU & 25 & 22 & & 1907 & 437 \\
CHO & 109 & 43 & & $22660^{*} \dagger$ & 3142 \\
CON & 30 & 16 & & 32 & 328 \\
\hline
\end{tabular}

$\mathrm{GLU}+\mathrm{CHO}$, glutamate + carbohydrate trial; $\mathrm{GLU}$, glutamate only trial; $\mathrm{CHO}$, carbohydrate only trial; CON, control trial.

* Mean value was significantly different from that of the CON trial. † Mean value was significantly different from that of the GLU trial. 


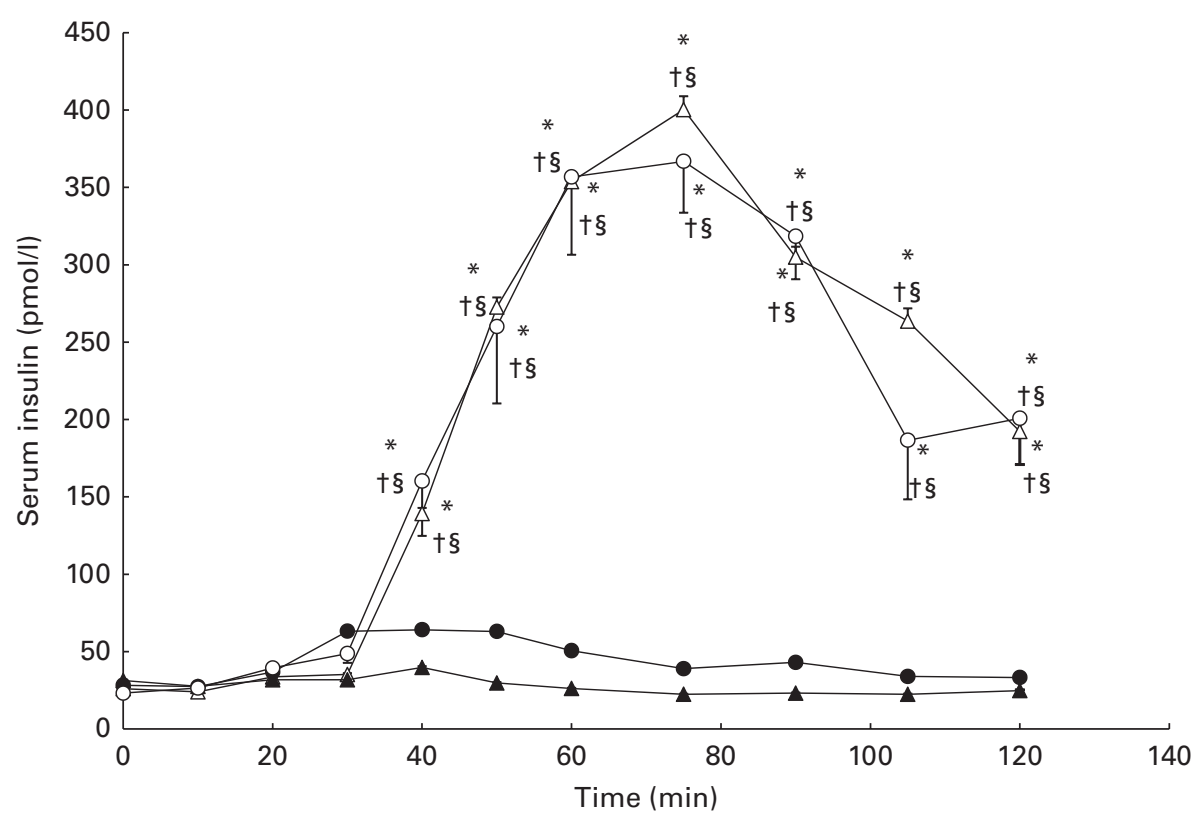

Fig. 3. Serum insulin concentrations during the glutamate + carbohydrate (GLU $+\mathrm{CHO}$; $)$, glutamate only (GLU; $\bullet$ ), carbohydrate only (CHO; $\Delta$ ) and control $(\mathrm{CON} ; \mathbf{\Lambda})$ trials. Values are means, with their standard errors represented by vertical bars. ${ }^{*}$ Mean value was significantly different from fasting values within each trial $(P<0.05)$. † Mean value was significantly different from that of the CON trial $(P<0.05)$. $\S$ Mean value was significantly different from that of the GLU trial $(P<0.05)$. Due to technical problems in blood draws, $n 8$ for time points $10-50$ and $75-120 \mathrm{~min}$ in the CON trial.

\section{NEFA and glycerol}

Serum NEFA concentrations decreased significantly from baseline during the GLU trial, reaching a nadir of $0 \cdot 20$ (SE 11.0 ) $\mathrm{mmol} / \mathrm{l}$ at $90 \mathrm{~min}$. However, serum NEFA concentrations returned to baseline levels $(0.49(\operatorname{se~} 0.30) \mathrm{mmol} / \mathrm{l})$ at $120 \mathrm{~min}$. No significant differences from the CON trial were observed. During the $\mathrm{GLU}+\mathrm{CHO}$ and $\mathrm{CHO}$ trials, we observed a more pronounced and prolonged decrease in NEFA concentrations at baseline from 50 to $120 \mathrm{~min}$ (Fig. 5). Between 90 and 105 min following MSG/placebo ingestion,
NEFA concentrations in both the $\mathrm{GLU}+\mathrm{CHO}$ and $\mathrm{CHO}$ trials were significantly lower than those in the CON trial (Fig. 5). We did not observe any changes in glycerol in any of the four experimental conditions.

\section{Discussion}

In the present study, we administered glutamate in the form of MSG, along with a carbohydrate load that was delayed $30 \mathrm{~min}$ relative to glutamate ingestion and we achieved peak plasma

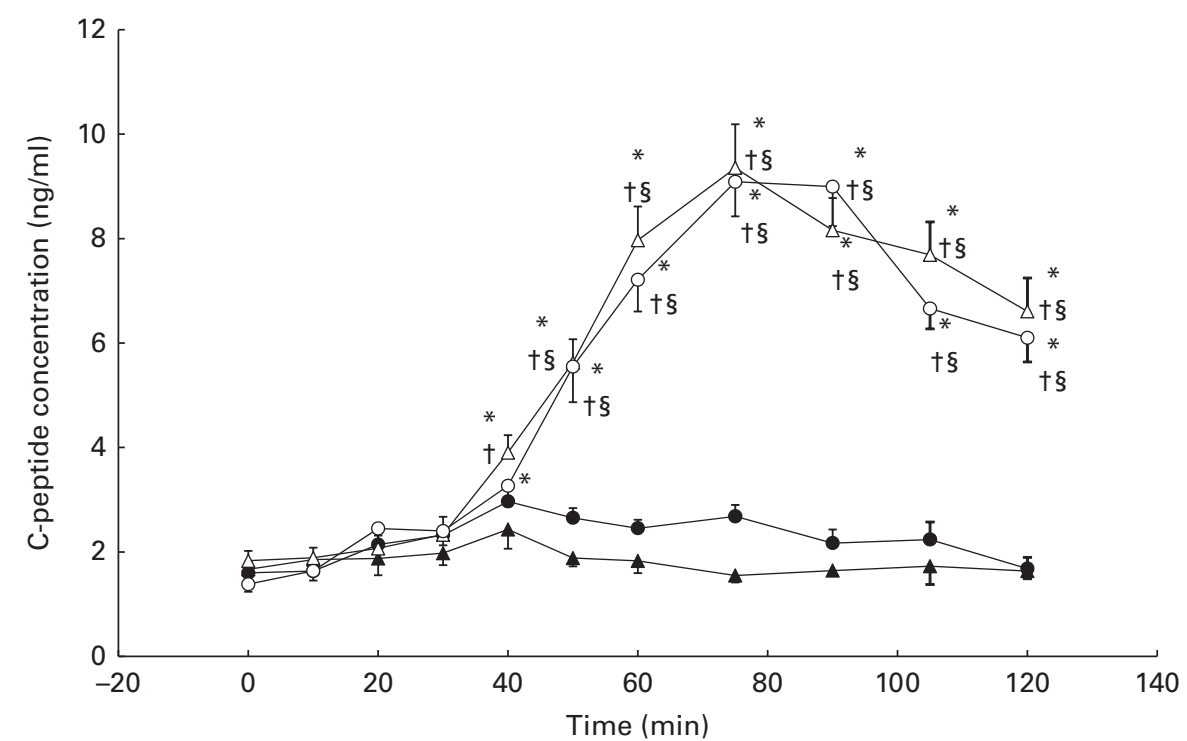

Fig. 4. Serum c-peptide during the glutamate + carbohydrate $(\mathrm{GLU}+\mathrm{CHO} ; \bigcirc)$, glutamate only $(\mathrm{GLU} ; \bullet)$, carbohydrate only $(\mathrm{CHO} ; \Delta)$ and control $(\mathrm{CON} ; \mathbf{\Delta})$ trials Values are means, with their standard errors represented by vertical bars. ${ }^{*}$ Mean value was significantly different from fasting values within each trial $(P<0.05)$. $\dagger$ Mean value was significantly different from that of the CON trial $(P<0.05)$. $\S$ Mean value was significantly different from that of the GLU trial $(P<0.05)$. Due to technical problems in blood draws, $n 8$ from time $75-120$ min in the CON trial. 


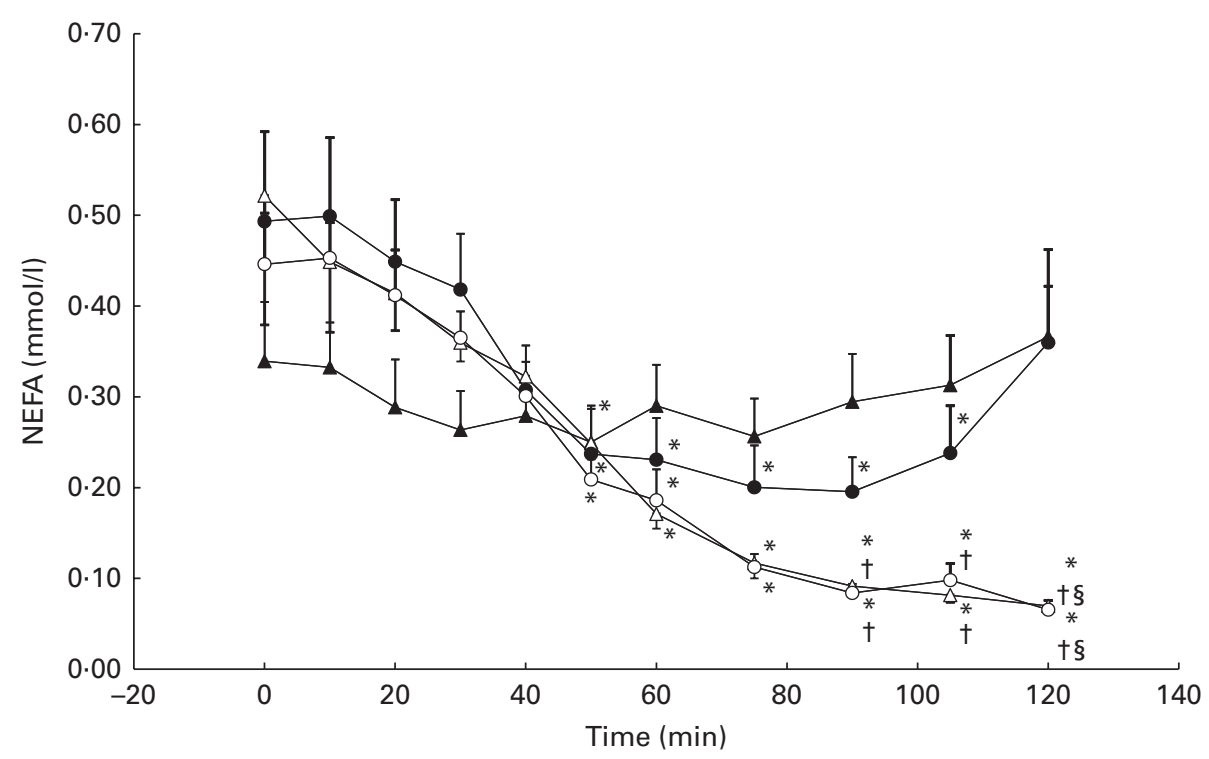

Fig. 5. Serum NEFA during the glutamate + carbohydrate (GLU + CHO; O), glutamate only (GLU; $\bullet$ ), carbohydrate only $(\mathrm{CHO} ; \Delta)$ and control (CON; $\mathbf{\Lambda})$ trials. Values are means, with their standard errors represented by vertical bars. ${ }^{*}$ Mean value was significantly different from fasting values within each trial $(P<0 \cdot 05)$. † Mean value was significantly different from that of the $\mathrm{CHO}$ trial $(P<0.05)$. $\S$ Mean value was significantly different from that of the GLU trial $(P<0.05)$. Due to technical problems in blood draws, $n 8$ from time $75-120 \mathrm{~min}$ in the CON trial.

glutamate and glucose concentrations in a similar time frame. We observed significantly elevated plasma glutamate concentrations relative to baseline values during the GLU trial (approximately 10-fold increase) as well as during the GLU + CHO trial (approximately 6-fold increase). The glucose response to a carbohydrate load was blunted when glutamate was increased in the circulation. Although we observed depressed serum glucose concentrations and delayed peak glucose concentrations during the GLU + CHO trial compared with the $\mathrm{CHO}$ trial, the average insulin and c-peptide responses were identical between these two conditions, suggesting that peripheral uptake of glucose may have been enhanced.

\section{Concurrent peak plasma glutamate and glucose concentrations were achieved with this protocol}

We successfully increased plasma glutamate concentrations by approximately 10-fold in the GLU trial and approximately 6-fold in the GLU + CHO trial via oral supplementation. Further, the observed increase in plasma glutamate concentrations was similar to that reported in previous studies that employed the same MSG dosage $(150 \mathrm{mg} / \mathrm{kg}$ body mass $)^{(7,12)}$. In comparison, Stegink et al. ${ }^{(13)}$ reported increases in plasma glutamate concentrations of only 2-fold when they administered MSG and carbohydrate at the same time. Thus, by staggering the administration of MSG and carbohydrate, we successfully achieved a simultaneous and significant increase in the availability of both glutamate and carbohydrate. An increase in plasma glutamate concentrations of this magnitude, when carbohydrate is also ingested, has not previously been achieved by any other group. Glutamate is typically sequestered by the intestines at the first pass ${ }^{(20)}$ and is oxidised by various reactions, the by-products of which are typically released into the portal circulation ${ }^{(21)}$. This process would be enhanced with simultaneous carbohydrate and glutamate ingestion, by providing pyruvate and glutamate as substrates in the alanine aminotransferase reaction. The staggered approach used in the present study probably reduced the sequestration of glutamate at the gut during carbohydrate ingestion, since the majority of the bolus of glutamate would have overwhelmed the pathways in the gut and have already entered into the circulation before the ingestion of carbohydrate.

\section{Glutamate is associated with improved glucose metabolism}

During the GLU + CHO trial, peak serum glucose concentration was, on average, depressed by $30 \%$, and delayed by $15 \mathrm{~min}$, relative to the $\mathrm{CHO}$ trial. Bertrand et al. ${ }^{(9)}$ reported similar findings in normal rats: glutamate was responsible for a significant decrease in glucose AUC during intravenous glucose tolerance tests and oral glucose tolerance tests. However, the peak value and timing of serum glucose concentrations was not affected by glutamate. Chevassus et al. ${ }^{(11)}$ administered glutamate during oral glucose tolerance tests in human subjects and noted no change in serum glucose levels. To our knowledge, these are the only studies that have assessed the response of serum glucose levels to glutamate ingestion. Although Stegink et al. ${ }^{(13)}$ administered glutamate and carbohydrate in their protocol, they only measured plasma glutamate levels. It is possible that we observed a greater dampening effect of glutamate on serum glucose in the present study due to the staggered administration of MSG and carbohydrate. Some authors have speculated that when MSG and carbohydrate are ingested simultaneously, glutamate is retained in the gut and prevented from entering into the circulation due to increased flux through the alanine aminotransferase reaction. Delaying carbohydrate administration in 
the present study, and therefore decreasing the availability of pyruvate (a substrate for the alanine aminotransferase reaction), might have allowed glutamate to enter into the circulation more efficiently. It may be that as circulating glutamate is taken up by skeletal muscle, it facilitates the simultaneous uptake of glucose by skeletal muscle. This hypothesis is supported by Thomassen et al. ${ }^{(22)}$ who conducted a study on patients who underwent arterial-femoral vein catheterisation and received intravenous MSG. As glutamate was infused directly into the circulation of patients, substrate utilisation in lower-limb skeletal muscle appeared to shift from NEFA to glucose. If glutamate facilitates glucose uptake by skeletal muscle, it does not necessarily follow that this increased glucose uptake would be captured using the Matsuda index. We did not observe differences in the Matsuda index of insulin sensitivity between the $\mathrm{CHO}$ and GLU + CHO trials; however, this index provides an assessment of whole-body insulin sensitivity and therefore may not be reflective of changes in peripheral tissues such as skeletal muscle. Moreover, the Matsuda index is dependent on changes in both insulin and glucose during a glucose challenge. Using the protocol in the present study, more invasive and informative techniques, such as muscle biopsies, are needed to further elucidate the role of glutamate in glucose metabolism.

Contrary to our hypotheses, insulin secretion was not on average augmented in the GLU + CHO trial compared with the $\mathrm{CHO}$ trial. Based on our sub-analysis, it appears that glutamate, when combined with carbohydrate, elicits one of two insulin responses, depending on the individual. Although the present study was not designed to explore this question, we did remark that the differences between these two groups of participants were not explained by factors associated with glucose handling, such as habitual activity levels (assessed using the Baecke questionnaire) ${ }^{(23)}$, fasting glucose $^{(24)}$, available glutamate ${ }^{(9)}$ or insulin sensitivity ${ }^{(19)}$. Further investigation into the cause of this difference in insulin response is warranted.

\section{Conclusions}

In delaying carbohydrate administration $30 \mathrm{~min}$ relative to MSG ingestion, we achieved peak plasma glutamate and carbohydrate concentrations within a similar time frame and magnitude relative to the consumption of MSG alone. Increased glutamate availability was associated with a blunted and delayed elevation in serum glucose concentrations without an accompanying increase in insulin secretion. MSG and carbohydrate supplementation therefore can be used as a tool to manipulate plasma glutamate concentrations, provided their administration is staggered. Future studies should investigate responses to glutamate and carbohydrate supplementation in muscle, while controlling for potential differences in insulin responses.

\section{Acknowledgements}

The present study was supported by the Natural Sciences and Engineering Research Council of Canada. The authors would like to thank Raja Panjwani and Eric Earl for their technical contributions as well as Erin Harvey for her statistical consultations. The authors' contributions are as follows: M. M. designed the study; K. M. D. S., M. M. and A. W. recruited the participants and collected the data; K. M. D. S., K. E. B., T. B., T. P. and A. W. performed the biochemical analysis; T. B. and K. E. B. completed the statistical analysis; K. M. D. S., K. E. B., T. B. and M. M. wrote the manuscript. All authors read, reviewed and approved the final manuscript. The authors declare no conflict of interest.

\section{References}

1. Gibala MJ, MacLean DA, Graham TE, et al. (1997) Anaplerotic processes in human skeletal muscle during brief dynamic exercise. J Physiol 502, 703-713.

2. Graham TE, Sgro V, Friars D, et al. (1998) Ammonia and amino acid metabolism in skeletal muscle: human, rodent and canine models. Med Sci Sports Exerc 30, 34-46.

3. Graham TE, Turcotte L, Kiens B, et al. (1997) Effect of endurance training on ammonia and amino acid metabolism in humans. Med Sci Sports Exerc 29, 646-653.

4. Mourtzakis M \& Graham TE (2002) Glutamate ingestion and its effects at rest and during exercise in humans. $J$ Appl Physiol 93, 1251-1259.

5. Mourtzakis M (2006) Carbohydrate metabolism during prolonged exercise and recovery: interactions between pyruvate dehydrogenase, fatty acids, and amino acids. J Appl Physiol 100, 1822-1830.

6. Mourtzakis M, Graham TE, Gonzalez-Alonso J, et al. (2008) Glutamate availability is important in intramuscular amino acid metabolism and TCA cycle intermediates but does not affect peak oxidative metabolism. J Appl Physiol 105, 547-554.

7. Graham TE, Sgro V, Friars D, et al. (2000) Glutamate ingestion: the plasma and muscle free amino acid pools of resting humans. Am J Physiol Endocrinol Metab 278, E83-E89.

8. Thomassen A, Bøtker HE, Nielsen TT, et al. (1990) Effects of glutamate on exercise tolerance and circulating substrate levels in stable angina pectoris. Am J Cardiol 65, 173-178.

9. Bertrand G, Puech R, Loubatieres-Mariani M, et al. (1995) Glutamate stimulates insulin secretion and improves glucose tolerance in rats. Am J Physiol Endocrinol Metab 269, E551-E556.

10. Bertrand G, Ravier M, Puech R, et al. (1997) Effects of glutamate on glucose tolerance and insulin secretion in a rat model of type II diabetes. Diabetologia 40, A132.

11. Chevassus H, Renard E, Bertrand G, et al. (2002) Effects of oral monosodium (L)-glutamate on insulin secretion and glucose tolerance in healthy volunteers. Br J Clin Pharmacol 53, 641-643.

12. Stegink LD, Filer L \& Baker G (1983) Effect of carbohydrate on plasma and erythrocyte glutamate levels in humans ingesting large doses of monosodium L-glutamate in water. Am J Clin Nutr 37, 961-968.

13. Stegink LD, Baker GL \& Filer LJ (1983) Modulating effect of Sustagen on plasma glutamate concentration in humans ingesting monosodium L-glutamate. Am J Clin Nutr 37, 194-200.

14. Petrie HJ, Chown SE, Belfie LM, et al. (2004) Caffeine ingestion increases the insulin response to an oral-glucosetolerance test in obese men before and after weight loss. Am J Clin Nutr 80, 22-28. 
15. Heinrikson RL \& Meredith SC (1984) Amino acid analysis by reverse-phase high-performance liquid chromatography: precolumn derivatization with phenylisothiocyanate. Anal Biochem 136, 65-74.

16. Lowry O \& Passoneau J (1972) A Flexible System of Enzymatic Analysis. New York: Academic Press pp. 146-218.

17. Duncombe WG (1964) The colorimetric micro-determination of non-esterified fatty acids in plasma. Clin Chim Acta 9, 122-125.

18. Allison D, Paultre F, Maggio C, et al. (1995) The use of areas under curves in diabetes research. Diabetes 18, 245-250.

19. Matsuda M \& Defronzo RA (1999) Insulin sensitivity indices obtained from oral glucose tolerance testing: comparison with the euglycemic insulin clamp. Diabetes Care 22, 1462-1470.

20. Matthews DE, Marano MA \& Campbell RG (1993) Splanchnic bed utilization of glutamine and glutamic acid in humans. Am J Physiol 264, 848-854.
21. Burrin DG \& Stoll B (2009) Metabolic fate and function of dietary glutamate in the gut. Am J Clin Nutr 90 , $850 \mathrm{~S}-856 \mathrm{~S}$

22. Thomassen A, Nielson TT, Bagger JP, et al. (1991) Effects of intravenous glutamate on substrate availability and utilization across the human heart and leg. Metab Clin Exp 40 , 378-384.

23. Petkovic-Koscal M, Damjanov V \& Djonovic N (2012) Influence of moderate physical activity on the levels of plasma lipoproteins in subjects with impaired glucose tolerance. Srp Arb Celok Lek 140, 51-57.

24. Hanefeld M, Koehler C, Fuecker K, et al. (2003) Insulin secretion and insulin sensitivity pattern is different in isolated impaired glucose tolerance and impaired fasting glucose: the risk factor in Impaired Glucose Tolerance for Atherosclerosis and Diabetes Study. Diabetes Care 26, 868-874 